\title{
Sintering behaviour of cemented tungsten carbide and steel bilayer
}

\begin{abstract}
The target of processing bimaterial components by cost effective powder metallurgy is to combine unique properties from different parts of the component. Differential shrinkage rate and more often, radial mismatch which is more consistent in bilayer owing to sintering conditions (time, rate, temperature etc.) is known to compromise the interfacial bond strength and integrity of these components. Therefore, the aim of this study is to analyze the sintering compatibility and evaluate the mismatch strain in cemented tungsten carbide (WC-Fe-C) and steel (Fe-W-C) bilayer processed through powder metallurgy (PM) to combine the hardness and toughness properties applicable in machining industries for drilling tools. Through geometrical measurement, mismatch strain rate between layers at different sintering temperatures was calculated and a value as low as $13.7 \%$ was observed at the interfacial zone of $\mathrm{MC}-0.2$ specimen sintered at $1280^{\circ} \mathrm{C}$ indicating a stronger bond between layers compared to those sintered at $1290^{\circ} \mathrm{C}$ and $1295^{\circ} \mathrm{C}$ where a huge mismatch was found increasing the tendency for delamination.
\end{abstract}

Keyword: Bilayer; Carbon content; Mismatch strain; Powder metallurgy 